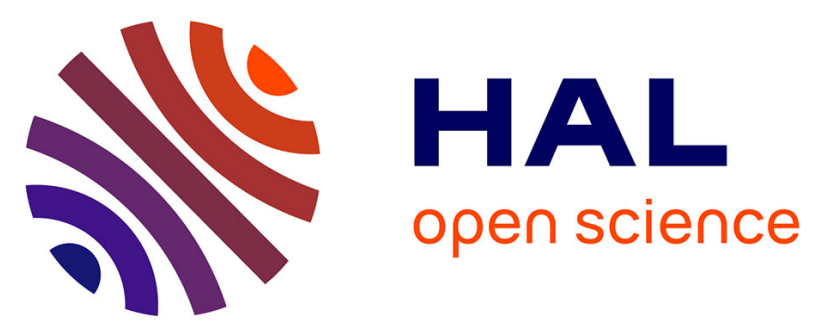

\title{
How important is consent in maternal serum screening for Down syndrome in France? Information and consent evaluation in maternal serum screening for Down syndrome: a French study.
}

Romain Favre, Nathalie Duchange, Christophe Vayssière, Monique Kohler, Nicole Bouffard, Marie-Christine Hunsinger, Anne Kohler, Cécile Mager, Muriel Neumann, Christine Vayssière, et al.

\section{- To cite this version:}

Romain Favre, Nathalie Duchange, Christophe Vayssière, Monique Kohler, Nicole Bouffard, et al.. How important is consent in maternal serum screening for Down syndrome in France? Information and consent evaluation in maternal serum screening for Down syndrome: a French study.. Prenat Diagn, 2007, 27 (3), pp.197-205. 10.1002/pd.1656 . inserm-00126553

HAL Id: inserm-00126553 https://www.hal.inserm.fr/inserm-00126553

Submitted on 4 Sep 2009

HAL is a multi-disciplinary open access archive for the deposit and dissemination of scientific research documents, whether they are published or not. The documents may come from teaching and research institutions in France or abroad, or from public or private research centers.
L'archive ouverte pluridisciplinaire HAL, est destinée au dépôt et à la diffusion de documents scientifiques de niveau recherche, publiés ou non, émanant des établissements d'enseignement et de recherche français ou étrangers, des laboratoires publics ou privés. 


\section{How important is consent in maternal serum screening for Down syndrome in France?}

\section{Information and consent evaluation in maternal serum screening for Down syndrome: a French study}

\section{Authors}

Romain Favre, MD *

Nathalie Duchange, $\mathrm{PhD} * *$

Christophe Vayssière, MD *

Monique Kohler, MD *

Nicole Bouffard *

Marie-Christine Hunsinger *

Anne Kohler*

Cécile Mager *

Muriel Neumann *

Christine Vayssière *

Brigitte Viville, MD*

Christian Hervé, MD **

Grégoire Moutel, MD **

* Département d'échographie et de Médecine fœtale. 19 rue Louis Pasteur, CMCOSIHCUS. Schiltigheim / Strasbourg France.

** Laboratoire d'éthique médicale et médecine légale, Faculté de médecine, Université Paris 5, 45 rue des Saints Pères, 75006 Paris \& Institut International de Recherche en Ethique Biomédicale (IIREB).

Key words: Prenatal diagnosis, Down syndrome, maternal serum screening, informed consent.

Corresponding author: Romain Favre. 19 rue Louis Pasteur. CMCO-SIHCUS. Schiltigheim/Strasbourg. France.

Email: romain.favre3@wanadoo.fr 


\section{Abstract}

Objectives. We evaluated the level of information and informed consent for maternal serum screening (MSS) for Down syndrome (DS) in the second trimester of pregnancy.

Methods. We studied the population of pregnant women attending obstetric consultations in two French hospitals over a three-month period. The women were assigned to three groups according to MSS results for DS: women at high risk of having a child with DS (group 1), women at low risk (group 2) and women who did not undergo the test (group 3). A questionnaire was completed before the medical consultation, to assess the quality of consent before amniocentesis for the group at high risk and before the second-trimester ultrasound scan for the other two groups.

Results. We analysed 305 questionnaires for 89, 137 and 79 women belonging to groups 1, 2 and 3 respectively. In total, 123 women ( $40.3 \%$ [IC $95 \%, 35$ to $46 \%$ ]) were considered to be well informed; 33 (10\%, [IC $95 \% 8$ to $12 \%$ ]) had a high level of knowledge, but made choices not consistent with their stated attitude, whereas 149 (49.7\% [IC $95 \%, 45$ to $56 \%$ ]) were considered uninformed. Logistic regression analysis showed that maternal consent depended on three independent components:

1. The score attributed to the doctor for information about MSS $(t=4.216, p<0.001)$.

2. Whether the patient belonged to group $1(\mathrm{t}=-2.631, \mathrm{p}<0.009)$.

3. Educational level (< high-school diploma, high-school diploma or at least two years of higher education after high school $)(\mathrm{t}=2.324, \mathrm{p}<0.02)$.

The rate of consent increased with educational level and was highest for the women in group 1 and for those whose doctor had a high information score.

Conclusions. Our findings clearly show that women are provided with insufficient information concerning MSS screening for DS in the second trimester of pregnancy for real and, valid consent to be obtained. 


\section{Introduction}

Published studies of prenatal screening (PS) for Down syndrome (DS) based on maternal serum markers have been based essentially on laboratory results. Few studies have assessed whether women really understand the information they are given (1). In 1993, the French National Ethics Consultative Committee (CCNE) made a number of specific recommendations concerning screening for DS and the use of genetic tests (2). Certain conditions must be respected, including, in particular:

- Intelligible and appropriate medical information should be provided for the woman before the test and the woman should be offered counselling if desired.

- The determination of maternal serum markers should be carried out in an accredited laboratory.

According to the CCNE, the prenatal diagnosis of Down syndrome is felt to be "a misfortune for the individual, an emotional ordeal and an economic burden for the family and society" This assertion highlights the immediately negative stigmatisation of Down syndrome, which imposes itself as fact. This view led to the decision to favour prenatal diagnosis rather than perinatal management. However, the CCNE specifically stated in its opinion that screening should not be approached as "a massive programme to eliminate Down syndrome, as such a programme would pose major problems."

Following the CCNE recommendations, three legal texts were issued concerning prenatal diagnosis. Firstly, a decree published in May 1995 (3) defined the conditions for PS as follows: - biological analyses should be preceded by a medical consultation, making it possible to provide patients with information about the disease concerned, the means of screening used and the expected results; - provision was also made for informing the patients about the inherent risks of the sampling process used for screening and the consequences of screening; - the doctor is required to sign an attestation certifying that he or she provided the pregnant woman with all this information. A second decree published in September 1997 (4) imposed a requirement for written consent from the patient, acknowledging the receipt of information. This document also addresses the possibility of detecting other abnormalities. Finally, a decree published in February 1999 (5) added three new obligations concerning:

- The laboratory: The laboratory responsible for karyotyping must be issued with the attestation concerning the medical consultation and the signed consent form from the 
patient, in application of the public health code. The results of the test must be transmitted to the woman by the prescribing doctor.

- Maternal serum screening: This test can be carried out only during the 15 th, 16 th, 17 th and 18th weeks of amenorrhoea.

- The results: The results of the test can be transmitted to the woman only by the prescribing doctor.

The legislation has thus positively integrated the recommendations of the CCNE and clearly sought to provide a rigorous framework for this screening. However, it remains unclear how well these recommendations are applied and whether the levels of information and consent conform to ethical requirements.

In this paper, we evaluated the level of knowledge and of informed consent of women at the time of prescription of MSS for DS screening. We addressed the following questions:

Was the level of medical information provided on the consequences of screening pertinent? Did it depend on the social environment and medical practices?

How can patients exercise their autonomy at the time of MSS prescription if the information provided is too complex or not detailed enough?

Depending on the results to these two questions, how valid is the consent obtained? 


\section{Methods}

\section{1) Study population}

We carried out a prospective study of pregnant women recruited from the fetal ultrasound and prenatal diagnosis departments of two hospital structures (the SIHCUS-CMCO and HUS, Strasbourg). The study was carried out over a three-month period, from June 1st to August 30th, 2003. All women consulting in these structures were asked to participate in the study. Inclusion was exhaustive and only foreign patients unable to understand French were excluded $(n=45)$. These patients were migrants and didn't understand the majority of the questions. The members of staff responsible for completing the questionnaire (medical secretaries and midwives) were trained to ensure that they could explain medical terms and answer questions from women, without influencing their response. The questionnaire was completed before the medical consultation, to assess the woman's level of knowledge. The women were assigned to three distinct groups classically compared in obstetric studies $(6,7)$ :

Group 1: women who underwent MSS who were at high risk of carrying a baby with Down syndrome (estimated risk of Down syndrome $>1 / 250$ ). These patients were included in the study during consultations at our centre for amniocentesis, between the 17 th and 20th weeks of amenorrhoea.

Group 2: women who underwent MSS but were at low risk of carrying a baby with Down syndrome. These women were included at the time of the second-trimester ultrasound scan, between the 19th and 23rd weeks of amenorrhoea.

Group 3: women who did not undergo MSS. These women were included at the time of the second-trimester ultrasound scan, between the 19th and 23rd weeks of amenorrhoea.

Using the Michie' study (10), we estimated that a minimum sample of 300 patients was required to detect a difference of $10 \%$ in rates of informed consent between the three groups with $80 \%$ power at the $5 \%$ level of significance. 


\section{2) Questionnaire}

Our methodology is based on the approach of Michie (8) and Marteau (9), allowing a multidimensional analysis of informed consent at three levels. This analysis, in addition to considering "knowledge", which is generally the only criterion used to assess the validity of consent, also integrates two other essential dimensions: personal attitude of the patient to the test (values) and choice (uptake). The questionnaire we developed is based on closed questions and is strictly anonymous (appendix I). The first part (questions 1 to 6) provides a brief description of the patient: age, level of education, first language spoken, number of children and obstetric history. The second part (questions 7 to 11) concerns the profile of the prescribing doctor: sex, age group and type of practice. The third part (questions 12 to 16) consists of 5 closed questions exploring the information provided and allowing the attribution of a score for the medical interview. The median is calculated to define a threshold below which the level of information is considered insufficient. Questions 17 to 26 evaluate the patient's knowledge by means of questions with "true or false" responses, giving a maximum score of 20 points. The median is again calculated to define a threshold below which the patient's knowledge is considered poor. Finally, questions 27 to 31 evaluate the attitude of the patients to the test. The questionnaire has been validated for comprehensibility with10 patients before being used for the study.

The analysis was carried out as described by Marteau and Michie (9,10,11). It was based on three criteria previously validated and adopted by scientific societies: the patients' level of knowledge, the personal attitude of each patient to screening (values) and the choice as to whether to undergo or to refuse the test. Patients can be classified into eight categories on the basis of these three criteria (Figure 1). Only groups 1 and 4 provide truly informed consent.

We used the following statistical tests: $\chi^{2}$ tests to compare individual variables between groups, Student's t-test and logistic regression to evaluate the relative importance of factors and the interactions between them, using a significance threshold of $p=0.05$ (SPSS 13.0). 


\section{Results}

During the study period, 350 pregnant women were included; 45 of the questionnaires could not be analysed as they were incomplete $(13.1 \%)$, giving a total of 305 questionnaires for the final analysis.

The women were assigned to three groups: Group 1, 89 patients (29.2\%); Group 2, 137 patients $(44.9 \%)$ and Group 3, 79 patients $(25.9 \%)$.

\section{General data}

General data are issued from questions 1 to 11 and concerned both the population of women who answered the questionnaire and the population of doctors that followed their pregnancy.

Mean maternal age was $30.7 \pm 4.8$ years $(18-42)$ for the entire study population, $31.9 \pm 4.2$ years for group $1,30 \pm 4.5$ years for group 2 and $30.6 \pm 5.5$ years for group 3 The difference between group 1 and the other two groups was significant $(\mathrm{p}<0.001)$. The mean number of children per woman was $0.77 \pm 0.96(0-5)$. The women in group 1 had significantly $(\mathrm{p}<0.01)$ more children than those in groups 2 and 3 (group 1, $1.02 \pm 1$; group $2,0.69 \pm 0.9$ and group 3, $0.61 \pm 0.68$ ). In total, 33 women had a history of abortion for medical reasons, and 65 had at least one miscarriage. No significant difference was found between the three groups for these two factors. The level of education of the women was distributed as follows: 83 (27\%) had not completed high school, 49 (16\%) had a high-school diploma and $173(56.7 \%)$ had completed at least two years of higher education after high school. No difference in educational level was found between the three groups.

The doctors following the pregnancy responsible for providing information about screening consisted of 153 men and 152 women. Nine patients were followed solely by midwives. No significant differences were observed between the three groups for these factors. The distribution of doctors according to type of practice was as follows: 227 patients $(74 \%)$ were seen by doctors in private practice and $78(26 \%)$ were seen by doctors practising in hospitals. Significantly fewer of the women in group 3 than in the two other groups were seen by doctors in private practice $(\mathrm{p}<0.0001)$. 


\section{Medical information about MSS}

In response to question 12 , which asked "Did the doctor talk to you about the use of serum markers for screening?", 197 women (64.6\%) answered affirmatively. The number of positive answers was significantly higher in group 1 than in groups 2 and 3 $(\mathrm{p}<0.001)$. No significant difference was found between groups 2 and 3 . It is interesting to note that $20 \%$ of women for group 1 and $44 \%$ for group 2 answered that they had not been informed about MSS although they underwent the test. Furthermore, 37\% of the patients in group 3 who did not undergo the test said that they had received no information about MSS. The mean duration of explanation was $9.9 \pm 7.8$ minutes for group $1,8.4 \pm 7.4$ minutes for group 2 and $6.6 \pm 4.6$ minutes for group 3 (Question 13). The difference in the mean duration of explanation for group 3 and that for the other two groups was close to the limits of statistical significance $(\mathrm{p}<0.05)$.

Question 14 explored the women' understanding of the screening test for those who had responded affirmatively to question $12(\mathrm{n}=197)$ : $140(71 \%)$ understood the approach well, 55 (28\%) had only partly understood the explanation, and $2(1 \%)$ said that they had not understood at all. No significant difference was found between the three groups.

Question 15 evaluated the number of patients in groups 1 and 2 who remembered having been given an explanatory document on MSS, considering that they all received such a document, in accordance with the decree of September $30^{\text {th }} 1997$ (1). Surprisingly, only $43 \%$ of the patients in group 1, and $36 \%$ of those in group 2, remembered having received this document. There was no significant difference between these two groups.

Question 16 concerned the obligatory/optional nature of MSS. In all three groups, one third of the patients thought that this screening test was obligatory.

For the score attributed to the doctor, the median ( 6 in this case) was defined as the threshold for considering the medical message to be satisfactory: 127 doctors (42\%) had a score below this threshold and only 178 (58\%) had a satisfactory score. The results for groups 1 and 3 were similar, but the scores for these two groups were significantly higher than those for group $2(\mathrm{p}<0.001)$.

\section{Women' level of knowledge concerning MSS}

Question 17 was designed to identify women capable of recognising that a risk "of $1 / 200$ is greater than a risk of 1/420". The correct response was obtained from 231 patients $(75.7 \%)$, with no difference between the groups. 
In response to question $18,43 \% \quad(n=133)$ thought that amniocentesis was obligatory if abnormal results were obtained for MSS.

Question 19 focused on the notion of "false-negative": "Can Down syndrome be excluded if the estimated risk is less than 1/600?". The correct response was given in $60 \%$ of cases. However, $40 \%$ of women thought that normal results for MSS meant that the baby could not have Down syndrome. Questions 20 and 21 evaluated the notions of "false-positive" and risk estimation. Correct responses concerning the notion of "falsepositive" were obtained from 92 patients (30\%). For risk estimation, $50.8 \%$ of women gave incorrect responses, over-representing the risk.

Questions 22 to 24 further evaluated women's knowledge: 49 (16\%) knew that MSS could also be used to screen for spina bifida, 52\% understood that MSS could be used to screen for other chromosome abnormalities and 95\% did not make a connection between the test and Klinefelter's syndrome.

In response to questions 25 and 26 respectively, 48 (15.7\%) replied that abortion was obligatory if Down syndrome was detected and $245(80 \%)$ were unaware that risk of miscarriage associated with amniocentesis could be up to twice the risk of Down syndrome.

No difference was found between the three groups for any of these questions. The median of the results obtained for these 10 questions was used to define the women's level of knowledge about MSS: the latter was higher in groups 1 and 3 than in group 2 $(\mathrm{p}<0.001)$. There was no significant difference between groups 1 and 3 .

\section{Maternal attitude to MSS}

Maternal attitude was evaluated through the answers to questions 27-30. The midpoint score for the results was 14 , which was taken as the threshold. As shown in Table I, 250 women $(82 \%)$ had a positive view of MSS. The differences between groups were highly significant. The women in group 2 had the most positive attitude, followed by those of group $1(\mathrm{p}<0.001)$ and group $3(\mathrm{p}<0.001)$ in which more than $40 \%$ of women were opposed to this test. However, it should be pointed out that $59.5 \%$ of the women in group 3 had a positive view of this test even though they declined to be tested.

\section{Global analysis: consent for MSS}

Global analysis of consent for MSS defined three levels (table II). In total, 123 women (40.3\% [IC $95 \%, 35-46 \%$ ]) could be considered to have given true informed consent. We 
found that $33(10 \%$ [IC $95 \% 8-12 \%]$ ) behaved in a manner inconsistent with their values, despite having a high level of knowledge. The rest of the population (149 patients; $49.7 \%$ [IC $95 \%, 45-56 \%$ ]) appeared to be poorly informed. The 115 women (12\%) falling into groups 6 and 7 in terms of the quality of consent had a low level of knowledge and did not behave in a manner consistent with their personal values.

All the variables assessed in the questionnaire were subjected to a logistic regression analysis, to identify those factors influencing consent. We found that the quality of consent for MSS depended entirely on three independent factors:

- The doctor's score for information about MSS $(\mathrm{t}=4.216, \mathrm{p}<0.001)$.

- $\quad$ Belonging to group $1(\mathrm{t}=-2.631, \mathrm{p}<0.009)$.

- $\quad$ Educational level $(\mathrm{t}=2.324, \mathrm{p}<0.02)$.

The quality of consent was the highest for patients belonging to group 1, with a high level of education, particularly if the doctor also had a high score. 


\section{Discussion}

\section{What role does consent play in practice?}

Consent is recognised to be an essential element of the doctor-patient relationship, but the condition for its validity is that it is supported by appropriate and comprehensible information. A quality of consent is also required to help patients to act in accordance with their own values.

The aim of this study was to explore the level of consent to screening for Down syndrome by maternal serum marker determination. We analysed the content of the information provided during the medical consultation preceding prenatal screening, focusing on elements that were previously recognized as essential (8): the information about Down syndrome, the significance of "low risk" and "high risk" results, the percentage of patients at low or at high risk, the risk of miscarriage following amniocentesis for patients at high risk, the percentage of patients at high risk who actually have a trisomic foetus, the possibility to detect other pathologies through the screening and finally, the information on the possibility of abortion for patients whose results demonstrate the presence of a Down syndrome in the foetus. These recommendations were adopted and adapted by the General Medical Council (12). In France, legal texts regulate the practice $(2,3,4,5)$, but scientific societies have not yet issued their own recommendations.

Efforts to improve the quality of information (content and approach) are essential, particularly as this has been a legal obligation since 2002 in France. Our results demonstrate the persistence of gaps in the information and consent process. They also show that a key element in the validity of consent is the quality of the medical interview, as evaluated by our score. Essential notions are poorly understood: $70 \%$ of our patients had a poor understanding of the notion of "false-positive" and 50.8\% evaluated the representation of risk poorly. Goel et al (17) highlighted the high level of anxiety associated with false-positive results and the false sense of reassurance in patients with false-negative results. The same trend has been observed by Gekas et al (6) and Smith et al (7) who reported that only $32 \%$ and $36 \%$ of women respectively understood the notion of "false-negative". Risk estimation was also poorly apprehended: $6 \%$ of women at high risk (group 1) thought that their child was trisomic and $21.5 \%$ of women at low risk (group 2) thought that the risk of having a trisomic child was as high as $50 \%$, whereas the risk remains less than $1 \%(6)$. Patients also had a very poor understanding of the notion of a 
"high risk group". In our study, only $30.2 \%$ patients understood this notion. Smith et al (7) reported that only $13 \%$ of patients understood this notion.

Information concerning other pathologies that can be possibly detected is also lacking in our study: $84 \%$ of women had never heard about spina bifida screening and 95.4\% were completely unaware of the existence of Klinefelter syndrome. This raises questions about how to inform about these issues. As for Down syndrome' screening, there are only administrative and medico legal forms concerning these pathologies, not integrated into a pedagogical medical approach. No educational or informational support was provided or discussed with the patient. This problem could be tackled by scientific societies, in collaboration with the representatives of parents' associations. The aim would be to inform women without worrying them.

The lack of prenatal information has already been highlighted by the work of Harris et al (13) dealing with the medical files of patients testing false-negative for Down syndrome. In another study, Lane et al (14) showed that only $16 \%$ of the doctors interviewed issued patients with a written document informing them about screening for Down syndrome. They also reported that $21 \%$ of midwives and $24 \%$ of obstetricians attested having received no specific recommendations.

Our results also show the difficulty for the patients to exert their autonomy as regard to the test. In our population, only $65 \%$ of the women declared that they received medical information about MSS. A similar study in the North of France showed that $57 \%$ of patients were not given the choice of accepting or refusing the test (6). Similarly, in England, 25\% of doctors prescribe these tests without providing any prior explanation (11). This could be due to a lack of formation of the professionals about these tests and their consequences, possibly accentuated by a lack of awareness of the need to inform patients $(15,16)$. This lack of knowledge leads to deficiencies in the advice provided by doctors (17).

Our findings should be read with cautions. The questionnaire is filled at different time between the three groups a long time after the medical counselling. Every patient should have a discussion with a healthcare professional in first trimester at 9 to 11 week's gestation. For the group 1, they will fill the questionnaire at a mid gestational age of 18 weeks before the amniocentesis. For group 2 and 3, it will be much later at $22-24$ week's of gestation at the time of the second morphological ultrasound. We can suppose that a great part of this discussion has been forgotten by the patients. Otherwise, patients from group 1 fill the questionnaire after having received the result of the MSS. For these 
women, it is not possible to say if the knowledge about the test and the consequences will not be discussed for the first time at this moment. If it is the case, the level of consent in group 1 should be much lower than what we observed. Finally, we decided to exclude 45 women issued from the migration. It is quite clear that these women have the worst situation in term of informed consent.

\section{Factors influencing consent}

Overall, $40 \%$ of patients consented to the screening of maternal serum markers: among these, $47 \%$ were partially informed and $12 \%$ were completely uninformed. The quality of consent for maternal serum screening depended on three independent factors tested in our study: educational level $(\mathrm{p}<0.003)$, belonging to group $1(\mathrm{p}<0.007)$ and doctor's score for the explanation of MSS $(\mathrm{p}<0.001)$.

Educational level had a significant effect on the quality of consent, suggesting that information should be modulated according to the profile of the patient. According to Braddock et al (19), advance in age and a low level of education are associated with a more dependent attitude. However, a close link between a high level of education and the probability of undergoing an invasive amniocentesis without first undergoing a screening test has been reported (21). This could be explained by the fact that highly educated women in socially comfortable situations have a greater demand for certainty as well as easier access to the health care system. Goel et al (17) clearly demonstrated the problem inherent in the language used by the patients. This point must clearly be taken into account, even though our study did not identify it as a determinant factor.

The doctor's score for the information given appears to be the most important of the three factors identified. It provides a cumulative estimate of all factors that can be used to assess the quality of the doctor-patient relationship during the interview preceding the prescription of serum markers. In our study, $35.4 \%$ of the patients reported that they had received no prior explanation. The patient's perception of the test as optional or obligatory was directly correlated with the doctor's score. We found that $30 \%$ of patients thought that the test was obligatory. The doctor's score was satisfactory for only $58 \%$ of the patients overall, being below $50 \%$ for group 2 and significantly lower in the other two groups. This highlights the importance of increasing the duration of the interview, providing a pedagogical document independent of the consent form and ensuring that the patient has understood the information. 


\section{Importance of consent and the stakes for public health}

A survey carried out in 1998 evaluating the information provided prior to consent, the transmission of the results and the subsequent patients' decision-making concluded that improvements were required (22). Nevertheless, no specific measures have been implemented since this time. In 2006, this unresolved issue has become a major public health concern.

As the age at which women become pregnant increases, so does the number of pregnancies affected by Down syndrome. French data show that the total prevalence of Down syndrome increased from 17 cases per 10,000 in 1990 to 26 cases per 10,000 in 2001 (23). The frequency of amniocentesis in Paris increased from 12 to $16 \%$ of pregnancies between 1995 and 1999 (23). The number of abortions of foetuses identified as having Down syndrome has also increased from 38.6\% in 1990 to $75.5 \%$ in 2001 (23). French public authorities have clearly chosen to increase access to prenatal diagnosis without improving the conditions for taking care of these children. Reflection on the management of this disability is essential in order to avoid automatic screening and its consequences. This is particularly important as the life expectancy of Down syndrome patients has increased from 20 to more than 50 years in less than 30 years. In the absence of debate on this issue, doctors are likely to seek to protect themselves legally, trying to prevent the birth of disabled children and any possibility of litigation with the parents. Since the Perruche Affair (24), a "safety-first" medicine has emerged in which professionals gradually slide towards the principle of precaution. 


\section{Conclusion}

The answer to the question "Is sufficient medical information provided to pregnant women about maternal serum screening?" is clearly "no". Based on our results, the overall level of knowledge of the women as regard to maternal serum screening for Down syndrome was low and too many of them considered the test to be obligatory. It is therefore difficult for patients to exercise their autonomy. The low level of women giving truly informed consent demonstrates that this screening does not, in practice, fulfil to the expectations formulated by politicians in the French legislation or to ethical principles.

There is currently a clear trend towards mass screening, as feared by the CCNE in 1993. 


\section{References}

1. Green J.M. Serum screening for Down's syndrome: experiences of obstetricians in England and Wales. B.M.J. 1994; 309: 769 - 72.

2. CCNE. Opinion $\mathrm{N}^{\circ} 37$. Opinion on the detection of the risk of foetal trisomy 21 by blood tests in pregnant women. Report.1993. www.ccne-ethique.fr

3. France. Decree No. 95-559 of 6 May 1995 concerning cytogenetic and biological analysis used for prenatal diagnosis in utero. Journal official nº8 (1995) 7362 . $\underline{\text { www.legifrance.gouv.fr }}$

4. France. Order of 30 september 1997 on the consent of pregnant women to the carrying out of the analyses referred to in article r. 162-16-1 of the public Health Code. Journal official n³1 (1997) 15820-15821. www.legifrance.gouv.fr

5. France. Order of 11 february 1999 modifying the order of 3 april 1985 establishing the Nomenclature for biological analysis. www.legifrance.gouv.fr

6. Gekas J., Gondry J., Mazur S., Cesbron P., Thepot F.. Informed consent to serum screening for Down Syndrome: Are women given adequate information? Prenat. Diagn. $1999 ; 19: 1$ - 7 .

7. Smith D.K., Shaw R.W., Marteau T.M.. Informed consent to undergo serum screening for Down's syndrome: The gap between policy and practice. BMJ 1994; 309: 776 - 7.

8. Michie S., Dormandy E., Marteau T.M. Informed choice: understanding knowledge in the context of screening uptake. Patient Education and Counseling. 2003; 50: 247 253.

9. Marteau T.M., Dormandy E., Michie S. A measure of informed choice. Health Expectations 2001; 4: 99 - 108. 
10. Michie S., Dormandy E., Marteau T.M. The multi-dimensional measure of informed choice: a validation study. Patient Education and Counseling. 2002; 48: (1) 87 - 91.

11. Michie S., Smith D., Marteau T.M. Prenatal tests: How are women deciding? Prenat. Diagn. $1999 ; 19: 743-48$

12. General Medical Council. Seeking patient's consent. The ethical considerations. London. GMC 1999. www.gmc-uk.org

13. Harris R., Lane B., Harris H., Williamson P., Dodge J., Modell B et al. National confidential enquiry into counselling for genetic disoders by non-geneticists: general recommandations and specific standards for improving care. Br. J. Obstet. Gynecol. 1999; 106: $658-663$.

14. Lane B., Challen K., Harris H.J., Harris R. Existence and quality of written antenatal screening policies in the United Kingdom: postal survey. BMJ 2001; 322(7277):22-3.

15. Sadler M. Serum screening for Down's syndrome: how much do health professionals know? Br. J. Obstet. Gynecol. 1997; 104 : 176 -179.

16. Dormandy E., Marteau T.M.. Uptake of prenatal screening test: the role of health care professionals' attitudes towards the test. Pren. Diagn. 2004; 24 : $864-868$.

17. Goel V., Glazier R., Holzapfel S., Pugh P., Summers A. Evaluating patient's knowledge of maternal serum screening. Prenat. Diagn. $1996 ; 16: 425$ - 30.

18. Favre R. [Screening for Down syndrome. Was informed consent realized? ]. DEA in Biological Sciences and Medical Ethics, Deontology and Medical Responsibility. 2004. www.ethique.inserm.fr

19. Braddock C.H., Fihn S.D., Levinson W., Jonsen A.R., Pearlman R.A. How doctors and patients discuss routine clinical decisions. J. Gen. Intern. Med. 1997; 12 (6): 339. 
20. World Medical Association declaration of Helsinki. Ethical principles for medical research involving human subjects. Helsinki June 1964. Modification Tokyo 2004. www.wma.net

21. Khnoshnood B., Blondel B., De Vigan C., Bréart G.. Effects of maternal age and education on the pattern of prenatal testing: Implications for the use of antenatal screening as a solution to the growing number of amniocentesis. Am. J. Obstet. Gynecol. 2003; 189: 5: $1336-1342$.

22. Seror V., Costet N., Aymé S.. [Information disclosure when screening for trisomy 21: a decision-making aid for pregnant women?] J. Gynecol. Obstet. Biol. Reprod. 2000 ; $29: 492-500$.

23. Goujard J. [Are there any changes in Down Syndrome prevalence in France following the implementation of the measurement of nuchal translucency and maternal serum screening?] Gynecol. Obstet. Fertil. 2004 ; 32 : 496 - 501.

24. Moutel G., Francois I., Moutard M-L., Herve C. [The Perruce decree, an opportunity to question the acceptance of a handicap and the relationship between physicians, justice and society]. Presse Med. $2002 ; 31: 632-635$. 
Appendix I. Questionnaire (18)

Did you have a blood test to screen for Down syndrome?

$$
\text { Yes - No }
$$

If yes, was the result

Normal - Abnormal?

1. How old are you?

2. What is your level of education? No high-school diploma - High-school diploma - at least 2 years of further education after high school

3. What is your first language? French - Other

4. How many children do you have?

5. Have you ever had an abortion? Yes - No

6. Have you ever had a miscarriage? Yes - No

\section{$\underline{\text { Prescribing doctor }}$}

7. Is the doctor following your pregnancy a Man - Woman?

8. Is your doctor a midwife?

Yes - No

9. How old is he or she?

Under 50 - Over 50.

10. Where does he or she work?

Hospital - Private

11. If your doctor works at a hospital, is it a university hospital? Yes - No 


\section{Second-trimester maternal serum screening}

12. Did the doctor inform you about the use of serum markers for screening during the second trimester? Yes - No.

13. How long did the explanation last? minutes.

14. Did you understand this explanation? No - Partly - Yes

15. Did the doctor give you an explanatory document? Yes - No

16. Is this blood test obligatory?

Yes - No - I don't know

Which of the following statements are true? Circle the correct response.

17. A risk of Down syndrome of $\mathbf{1 / 2 0 0}$ is lower than a risk of $\mathbf{1 / 4 2 0}$.

True False

18. Amniocentesis is obligatory if the risk is higher than $\mathbf{1 / 2 5 0}$.

True False

19. Down syndrome is excluded if the risk of Down syndrome is lower than $1 / 600$.

True False

20. If $\mathbf{1 0 0}$ patients decide to undergo maternal serum screening, how many will have an abnormal test result?

$$
5 \%-15 \%-20 \%-80 \% \text {. }
$$

21. If a patient has an estimated risk of $\mathbf{1 / 5 0 ,}$ what is the probability that the foetus has Down syndrome?

$20 \%-10 \%-2 \%$. 
22. Serum markers can also be used to screen for spina bifida.

True False

23. If you have an amniocentesis, only chromosome 21 will be analysed.

True False

24. Your doctor discussed the possibility of diagnosing Klinefelter syndrome.

True False

25. Abortion is obligatory if the results confirm the diagnosis of Down syndrome.

True False

26. If you have a risk of Down syndrome of $1 / 250$, the risk of a miscarriage linked to amniocentesis is twice as high. $\quad$ True False

For the following questions, circle the appropriate number between 1 and 7. For example, if you feel that having a screening test is a very good thing, you should circle number 7 in question 29. Conversely, if you feel that such screening is highly unpleasant, you should circle number 1 in question 30. If you feel that this screening is moderately worrying, you should circle number 3 in question 27.

I feel that screening for Down syndrome by maternal serum screening in the second trimester of pregnancy (15 to 17 weeks into the pregnancy) is:

27. Worrying

$$
1-2-3-4-5-6-7 \text {. Reassuring }
$$

28. Pointless

$1-2-3-4-5-6-7$. Useful

29. A bad thing

$1-2-3-4-5-6-7$. A good thing

30. Unpleasant

$1-2-3-4-5-6-7 . \quad$ Pleasant

31. Which of these statements best applies to you. Tick the appropriate box.

I feel that maternal serum screening in the second trimester of pregnancy is a good idea; I am the type of person who likes to have all the tests I can.

I don't feel that maternal serum screening in the second trimester of pregnancy is a good idea; due to the potential consequences, I am not convinced that this test is important. 
Table I. Maternal attitude to maternal serum screening.

\begin{tabular}{|c|c|c|c|c|c|c|}
\hline \multirow{6}{*}{$\begin{array}{l}\text { Attitude to } \\
\text { maternal serun } \\
\text { screening }\end{array}$} & & & \multicolumn{3}{|c|}{ Group } & \multirow{2}{*}{ Total } \\
\hline & & & 1 & 2 & 3 & \\
\hline & \multirow{2}{*}{ Positive } & No. & 75 & 128 & 47 & 250 \\
\hline & & $\%$ & $84.3 \% * *$ & $93.4 \% * *$ & $59.5 \%$ & $82.0 \%$ \\
\hline & \multirow{2}{*}{ Negative } & No. & 14 & 9 & 32 & 55 \\
\hline & & $\%$ & $15.7 \%$ & $6.6 \%$ & $40.5 \%$ & $18.0 \%$ \\
\hline \multirow{2}{*}{\multicolumn{2}{|c|}{ Tc }} & No. & 89 & 137 & 79 & 305 \\
\hline & & $\%$ & $100.0 \%$ & $100.0 \%$ & $100.0 \%$ & $100.0 \%$ \\
\hline
\end{tabular}

** $\mathrm{p}<0.001$ 
Table II. Level of consent for maternal serum screening

\begin{tabular}{|c|c|c|c|c|c|}
\hline $\begin{array}{l}\text { Category according } \\
\text { to Marteau }\end{array}$ & Knowledge & Attitude & Test carried out & No. & Level of consent \\
\hline 1 & + & + & + & $\begin{array}{c}100 \\
32.79 \%\end{array}$ & \multirow{2}{*}{$\begin{array}{c}123 \\
(40.3 \%)\end{array}$} \\
\hline 4 & + & - & - & $\begin{array}{c}23 \\
7.54 \%\end{array}$ & \\
\hline 2 & + & - & + & $\begin{array}{c}11 \\
3.61 \%\end{array}$ & \multirow{2}{*}{$\begin{array}{c}33 \\
(10 \%)\end{array}$} \\
\hline 3 & + & + & - & $\begin{array}{c}22 \\
7.21 \%\end{array}$ & \\
\hline 5 & - & + & + & $\begin{array}{c}103 \\
33.77 \%\end{array}$ & \multirow{4}{*}{$\begin{array}{c}149 \\
(49.7 \%)\end{array}$} \\
\hline 6 & - & - & + & $\begin{array}{c}12 \\
3.93 \%\end{array}$ & \\
\hline 7 & - & + & - & $\begin{array}{c}25 \\
8.20 \%\end{array}$ & \\
\hline 8 & - & - & - & $\begin{array}{c}9 \\
2.95 \%\end{array}$ & \\
\hline Total & & & & 305 & \\
\hline
\end{tabular}




\section{Legend to Figure 1.}

Classifying choices, based on the three dimensions of knowledge, attitudes and uptake.

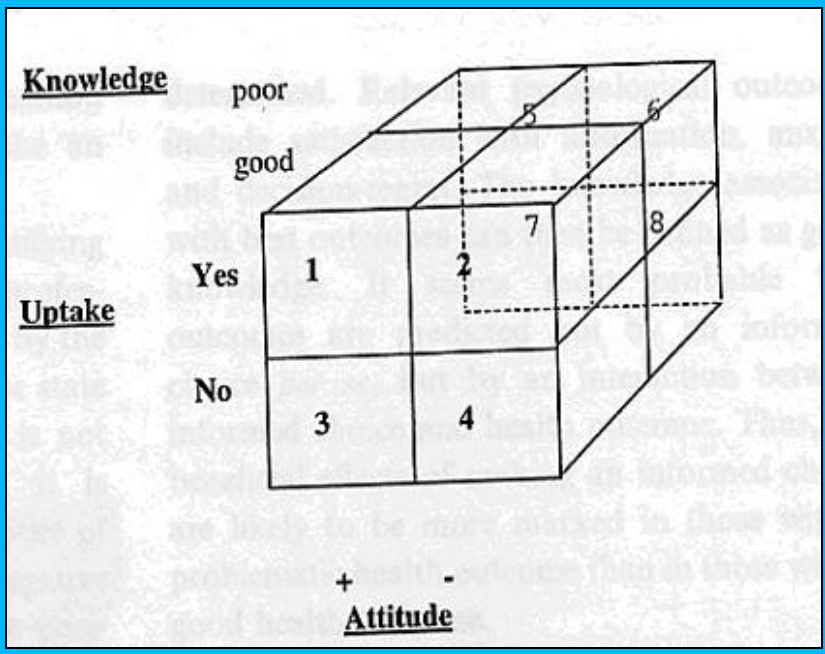

